\section{Adäquate Supportivtherapie macht eine gute Tumortherapie erst möglich}

«Prophylaxe geht vor Therapie» - unter dieser Maxime sollte heute die supportivtherapeutische Begleitung onkologischer Therapien erfolgen, wie Prof. Dr. med. Petra Feyer, Direktorin der Klinik für Strahlentherapie, Radioonkologie und Nuklearmedizin am Vivantes-Klinikum Neukölln/Berlin und Moderatorin des 17. Münchner Fachpresse-Workshops Supportivtherapie in der Onkologie ausführte. Ohne adäquate supportive Strategien, die sowohl die Prophylaxe und Therapie von Nebenwirkungen der Therapie als auch die Rehabilitation und zudem palliative Maßnahmen in inkurablen Situationen umfassen, sind die modernen onkologischen Therapiekonzepte nicht durchführbar. Dies verdeutlichen beispielhaft die in diesem Fachpresse-Workshop behandelten Themen aus der Supportivmedizin: Die konsequente antiemetische Prophylaxe ist eine wesentliche Voraussetzung für die Akzeptanz onkologischer Konzepte, die mit Übelkeit und Erbrechen assoziiert sind. Obwohl hier große Verbesserungen erreicht wurden, bestand bei den antiemetischen Schemata Optimierungsbedarf. Weiterentwicklungen, wie der nur einmalig pro Zyklus zu verabreichende NK1-Rezeptorantagonist Fosaprepitant, tragen deutlich zu weiteren Fortschritten in der Antiemese bei. Darüber hinaus verlangen viele Behandlungsansätze in der Onkologie per se oder zumindest bei Vorliegen zusätzlicher Risikofaktoren nach einer Primärprophylaxe der febrilen Neutropenie mit G-CSF, da ansonsten tödliche Komplikationen drohen. Als besonders wirkungsvoll hat sich in diesem Zusammenhang das Pegfilgrastim erwiesen, das nicht nur 1-mal pro Zyklus gegeben werden muss, sondern auch durch höhere Effek- tivität überzeugt. Ebenso ist die Schmerztherapie ein wichtiger Pfeiler der onkologischen Supportivtherapie, denn eine ausreichende Schmerztherapie ist die Voraussetzung für einen weitgehend selbstbestimmten Alltag von Tumorpatienten und sichert Lebensqualität. Hier spielt die rechtzeitige Gabe von starken Opioiden eine zentrale Rolle. Mit der Fixkombination aus retardiertem Oxycodon und retardiertem Naloxon steht heute ein stark wirksames und zugleich sehr gut verträgliches Opioid zur Verfügung.

17. Fachpresse-Workshop Supportivtherapie der POMMEmed GmbH am 07. Juli 2011 in München, unterstützt durch Amgen GmbH (München), GlaxoSmithkline GmbH (München) und Mundipharma GmbH, (Limburg)

Weitere Informationen bei

POMME-med GmbH

Dr. rer. nat. Petra Ortner

ortner@pomme-med.de
Conference at University Hospital Cologne

\section{Patient Participation at the Hospital} - Chances and Perspectives

Patient information is a prerequisite for patient participation and involvement. The results of a project to extend the supply of evidence based patient information were presented and discussed at a Conference at the University Hospital in Cologne on July 13th, 2011. More than 90 participants of both health care professionals as well as patient representatives joined the conference. This shows that there is a great demand for reliable, systematic patient information in the field of oncology. The conference was organized by the Centre of Integrated Oncology (CIO) at the University of Cologne/Bonn and the Institute of Health Economics and Clinical Epidemiology.
The main objectives of the project were a) to involve patients in the development of print media, b) to establish patient seminars on dealing with chemotherapy and management of cancer pain and c) to integrate information milestones into the care pathway. Patients' feedback on first draft of information leaflets was helpful to adapt wording and amount of text. Patients wanted precise and short summaries, as they are not capable of reading long texts when receiving cancer therapy. Patient seminars followed an interactive group approach, asking patients and family members to address their questions first and discuss every day matters within the group. Teamteaching was performed by physicians and nurses. Implementation of patient information into the care pathway can be systematized by defining significant events along the care pathway. We designed an out- line which can be used for various target groups.

Panel discussion with experts showed that patient information is a professional task in its own right and needs to become more visible and reliable. However, resources are needed to implement patient information into daily routine. Patients and self-help groups welcomed the commitment to improve patient information.

We acknowledge the support of the Dr. Werner Jackstädt Stiftung for funding the event.

Further Information

Universitätsklinikum Köln (AöR)

Dr. Stephanie Stock

Kerpener Straße 62, 50937 Köln

stephanie.stock@uk-koeln.de

\title{
PharmaTicker+++ PharmaTicker+++ PharmaTicker+++ PharmaTicker+++
}

Vifor Pharma. Bei Tumor-assoziiertem Eisenmangel normalisiert eine intravenöse Therapie mit Eisencarboxymaltose (ferinject ${ }^{\circledR}$ ) den Hämoglobin $(\mathrm{Hb})$-Wert. Dies ist das Ergebnis einer 12-wöchigen nicht-interventionellen Studie bei insgesamt 642 Krebspatienten mit absolutem oder funktionellem Eisenmangel. Durchschnittlich benötigten die Patienten $1333 \mathrm{mg}$ Eisen. Der größte Benefit wurde bei Patienten mit einem $\mathrm{Hb}$-Ausgangswert von unter $10 \mathrm{~g} / \mathrm{dl}$ erreicht.

Vifor Pharma Deutschland $\mathrm{GmbH}$

Dr. Stefanie Wesche

stefanie.wesche@ viforpharma.com
Janssen-Cilag. Die Therapie chronischer Schmerzen ist so individuell wie das Schmerzempfinden, die Schmerzintensität und das subjektive Behandlungsziel eines jeden Patienten. Daher komme der Auswahl des passenden Analgetikums ein hoher Stellenwert zu, so die einhellige Meinung der Experten auf dem 11. Zukunftsforum Schmerz des Unternehmens. Eine qualitätsgesicherte Schmerztherapie mit verlässlicher Schmerzlinderung und umfassender Verbesserung der individuellen Lebenssituation ermögliche z.B. Jurnista ${ }^{\circledR}$.

Janssen-Cilag GmbH jancil@its.jnj.com
Astellas Pharma. Seit 2002 hat sich Micafungin $\left(\right.$ Mycamine $^{\circledR}$ ) weltweit in der Behandlung systemischer Pilzinfektionen bewährt. Inzwischen wurde das Echinocandin bei mehr als $750000 \mathrm{~Pa}$ tienten eingesetzt. Eine retrospektive Analyse der Autoren Cornely et al. von 17 klinischen Studien mit Micafungin, die im März dieses Jahres in «Expert Opinion on Drug Safety» publiziert wurde, belegt das günstige Sicherheitsprofil der Substanz.

Astellas Pharma GmbH

Dr. Angela Gesing-Eisenbarth info@de.astellas.com 


\section{Das BOLERO-Studienprogramm:}

\section{RAD001 in der Therapie des metas- tasierten Mammakarzinoms}

Seit Dezember 2009 wird das Phase-IIIStudienprogramm BOLERO (Breast Cancer Trials of OraL EveROlimus) bei Patientinnen mit metastasiertem Mammakarzinom durchgeführt. BOLERO ist aktuell das größte internationale Studienprogramm, in dem ein mTORInhibitor zur Behandlung von lokal fortgeschrittenem oder metastasiertem Mammakarzinom untersucht wird.

In Deutschland wird jährlich bei rund 60000 Frauen Brustkrebs diagnostiziert. Über 50\% der Patientinnen entwickeln im Verlauf der Erkrankung Lokalrezidive oder Fernmetastasen. Zudem treten im Laufe der Behandlung oft Resistenzen gegenüber den verwendeten Wirkstoffen auf. Die Deregulation des mTOR-
Signalwegs ist entscheidend an der Tumorgenese des Mammakarzinoms beteiligt. RAD001 ist ein mTOR-Inhibitor, der in Phase-I/II-Studien bei Patientinnen mit metastasiertem Mammakarzinom bereits die über Crosstalks vermittelten Resistenzen auf HER2-zielgerichtete oder endokrine Therapien überwinden konnte. RAD001 hemmt das Schlüsselenzym mTOR, das als Regulator von Proliferation, Metabolismus und Angiogenese der Zelle eine zentrale Rolle bei der Bildung maligner Tumoren spielt.

\section{Das BOLERO-Studienprogramm}

Das Phase-III-Studienprogramm BOLERO untersucht die Wirkung von RAD001 bei HER2bzw. Estrogenrezeptor(ER)-positiven Mammakarzinomen bei fast 2000 Patientinnen weltweit - auch 30 deutsche Zentren sind beteiligt. Primärer Endpunkt aller BOLERO-Studien ist das progressionsfreie Überleben.

\section{Über RAD001}

RAD001 ist ein oral einzunehmender Inhibito von mTOR. Dieses Schlüsselenzym wirkt in der Tumorzelle als zentraler Regulator von Tumorzellwachstum, -teilung und -stoffwechsel sowie de Tumor-Angiogenese. Das Enzym wird durch das Angebot von Wachstumsfaktoren und Nährstoffen mittels Signaltransduktion reguliert. In malignen Tumoren wird mTOR oft überaktiviert. Dadurch kommt es zur vermehrten Proteinsynthese. Unkontrolliertes Wachstum, abnormer Metabolismus sowie vermehrte Tumor-Angiogenese werden dadurch ermöglicht und das Überleben der malignen Zellen wird gesichert. Durch die 1-mal tägliche Einnahme von RAD001 wird mTOR kontinuierlich gehemmt.

Weitere Informationen bei

Novartis Pharma GmbH

Dr. Irene Roth

Roonstraße 25, 90429 Nürnberg

Tel. +49 $911273-0$

www.novartisoncology.de
Bildung der Mikrotubuli hemmen, ohne diese zu verkürzen

\section{Eribulin - neue Perspektive beim metastasierten Brustkrebs}

Eine neue Behandlungsperspektive für Frauen mit metastasiertem Mammakarzinom eröffnet das neuartige Spindelgift Eribulin. Der Wirkstoff ist laut Dr. Joachim Bischoff, Magdeburg, indiziert zur Behandlung von Frauen mit Anthrazyklin- und Taxan-vorbehandeltem Karzinom, die bisher mindestens 2 Chemotherapien im fortgeschrittenen Stadium erhalten haben. «Es ist eine der ersten Substanzen, für die in dieser Situation Überlebensvorteile gegenüber dem Standardregime nachgewiesen werden konnten», so Bischoff bei der Jahrestagung der DGS in Dresden. Eribulin (Halaven ${ }^{\circledR}$ ) gehört zu den Tubulin-aktiven Zytostatika, unterschei- det sich in seinem Wirkprofil aber von anderen Tubulin-aktiven Wirkstoffen wie den Taxanen. Er hemmt vornehmlich das Wachstum der Mikrotubuli, unterbindet so deren Funktion und inhibiert damit die Zellteilung. Dagegen hat die Substanz im Unterschied zu anderen Tubulinaktiven Zytostatika keinen Einfluss auf die Verkürzung der Tubuli. Infolge des anderen Ansatzpunktes ist sie nach Bischoff auch bei einer Taxan-Resistenz wirksam.

In der Phase-III-Studie EMBRACE wurde gezeigt, dass die mit Eribulin behandelten Patientinnen gegenüber der Kontrollgruppe einen signifikanten Anstieg des Gesamtüberlebens aufweisen. 762 Frauen im Alter von 27 bis 85 Jahren, die durchschnittlich bereits 4 Chemotherapien erhalten hatten, wurden mit Eribulin als Monotherapie behandelt oder in der Kontrollgruppe mit einem Therapieverfahren nach Wahl des Arztes.
Die Analyse nach 422 Ereignissen ergab ein medianes Gesamtüberleben von 13,1 bzw. 10,6 Monaten (Hazard Ratio (HR) 0,81; p=0,041) [1]. «Es ist sehr erfreulich, dass wir nunmehr eine neue Substanz haben, mit der wir auch Frauen im fortgeschrittenen Stadium des metastasierten Mammakarzinoms noch eine Perspektive bieten können», so Professor Dr. Christian Jackisch, Offenbach.

Integrated Lecture der Session «Die metastasierte Situation», 31. Jahrestagung der DGS 2011, 24.06.2011, Dresden

Referenz

1 Cortes J, O‘Shaughnessy J, Loesch D et al.: Lancet 2011; 377:914-23.

Weitere Informationen bei

Eisai $\mathrm{GmbH}$

Adrienne Schmittat

Adrienne_Schmittat@eisai.net

\section{PharmaTicker+++ PharmaTicker+++ PharmaTicker+++ PharmaTicker+++}

Deutsche Krebsgesellschaft. Aktuell erkranken in Deutschland jährlich 57000 Frauen an Brustkrebs. Experten schätzen, dass etwa $1 / 3$ aller Brustkrebsfälle durch einen gesunden Lebensstil vermieden werden könnte. Daher gibt die Deutsche Krebsgesellschaft in Zusammenarbeit mit der Kooperationsgemeinschaft Mammographie einen Kalender im «Handtaschenformat» heraus, der Tipps zur Vorbeugung von Brustkrebs und auch die Möglichkeiten der Früherkennung zusammenfasst.

Pressestelle der Deutschen Krebsgesellschaft e.V André Franck

presse@krebsgesellschaft.de
Philips gab den Abschluss eines Vertrags zur Übernahme des Produktbereiches für Mammographie-Systeme von Sectra AB bekannt. Die Übernahme wird das Angebot von Philips im Bereich digitale Mammographie für hochwertige und strahlungsarme Brustbildgebung erweitern. «Die Investition in diese fortschrittliche digitale Mammographieplattform stellt einen wichtigen Schritt bei der Umsetzung der Strategie von Philips dar, sein Healthcare-Portfolio für Frauen zu erweitern", so Steve Rusckowski, CEO von Philips Healthcare.

Philips Deutschland GmbH Cornelia Rauchenberger cornelia.rauchenberger@ philips.com
Amgen. «Frauen mit Knochenmetastasen sind häufig unbehandelt», so Prof. Ingo Diel auf einer Pressekonferenz. «Dabei sind dies gerade die Patientinnen, die ärztliche Zuwendung in besonders starkem Maß benötigen.» Zumal zukünftig die Behandlung ossärer Komplikationen effektiver und einfacher mit dem 1-mal monatlich subkutan zu applizierenden RANKL-Inhibitor Denosumab erfolgen kann, der als XGEVA ${ }^{\circledR}$ seit dem 15. Juli 2011 von der EMA zur Prävention von skelettbezogenen Komplikationen bei Erwachsenen mit Knochenmetastasen aufgrund solider Tumoren zuglassen ist. Amgen $\mathrm{GmbH}$ Gerold Krischker gerold.krischker@amgen.com
KARGER ○ 2011 S. Karger GmbH, Freibur Verlag, Herausgeber, Redaktion und Verlagsgeschäftsführung übernehmen keine Verantwortung für den Inhalt dieser Rubrik. 